Article

\title{
Space Dimension Renormdynamics
}

\author{
Martin Bures ${ }^{1,2, *(D)}$ and Nugzar Makhaldiani ${ }^{1, *}$ \\ 1 Joint Institute for Nuclear Research, 141980 Dubna, Moscow Region, Russia \\ 2 Institute of Experimental and Applied Physics, Czech Technical University in Prague, Husova 240/5, \\ 11000 Prague 1, Czech Republic \\ * Correspondence: bures@physics.muni.cz (M.B.); mnv@jinr.ru (N.M.)
}

Received: 31 December 2019; Accepted: 15 April 2020; Published: 17 April 2020

\begin{abstract}
We aim to construct a potential better suited for studying quarkonium spectroscopy. We put the Cornell potential into a more geometrical setting by smoothly interpolating between the observed small and large distance behaviour of the quarkonium potential. We construct two physical models, where the number of spatial dimensions depends on scale: one for quarkonium with Cornell potential, another for unified field theories with one compactified dimension. We construct point charge potential for different dimensions of space. The same problem is studied using operator fractal calculus. We describe the quarkonium potential in terms of the point charge potential and identify the strong coupling fine structure constant dynamics. We formulate renormdynamics of the structure constant in terms of Hamiltonian dynamics and solve the corresponding motion equations by numerical and graphical methods, we find corresponding asymptotics. Potentials of a nonlinear extension of quantum mechanics are constructed. Such potentials are ingredients of space compactification problems. Mass parameter effects are motivated and estimated.
\end{abstract}

Keywords: space dimension dynamics; coulomb problem; extra dimensions

"... there will be no contradiction in our mind

if we assume that some natural forces are governed

by one special geometry, while other forces by another."

N. I. Lobachevsky

\section{Introduction}

Quarkonium spectroscopy indicates that between valence quarks inside hadrons, the potential on small scales has $D=3$ Coulomb form and at hadronic scales has $D=1$ Coulomb one (here we refer to Coulomb potential satisfying Gauss' law in $D$ dimensions, cf. Section 2 or [1]). We may combine this two types of behavior and form an effective potential in which at small scales dominates the $D=3$ component and at hadronic scale the $D=1$ component: the Coulomb-plus-linear potential (the "Cornell potential" [2]),

$$
V(r)=-\frac{k}{r}+\frac{r}{a^{2}}=\mu\left(x-\frac{k}{x}\right), \mu=1 / a=0.427 \mathrm{GeV}, x=\mu r,
$$

where $k=\frac{4}{3} \alpha_{s}=0.52=x_{0}^{2}, x_{0}=0.72$ and $a=2.34 \mathrm{GeV}^{-1}$ were chosen to fit the quarkonium spectra.

An important step in the solution of a theoretical problem is to find a good initial approximation in the corresponding mathematical model. Then by small deformations and a few terms in perturbation expansion we describe a physical phenomenon. When a deformation parameter (e.g., coupling constant) value increases, in some region the initial approximation might change into a new form. 
In the case of Quantum chromodynamics (QCD), the coupling constant increases with increasing distance between quarks, and in the intermediate region $(\sim 0.5 \mathrm{fm})$ the three dimensional hadronic space becomes a fractal - a space with intermediate dimension. At the hadronic scale $(\sim 1 \mathrm{fm})$, we again have a nice classical picture (one dimensional space) and already one gluon exchange between valence quarks gives a confining potential.

One of the main motivations for this work is to put the Cornell potential into a more geometrical setting. We aim to smoothly interpolate between the observed small and large distance behaviour of the quarkonium potential by considering dimension $D(r)$ of space of hadronic matter to be dynamically changing with $r$, i.e., the corresponding Coulomb potential is $V_{D}(r) \sim r^{2-D(r)}$, where the effective dimension of space $D(r)$ changes from 3 at small $r$ to 1 at hadronic scales $\sim 1 \mathrm{fm}$. In this paper, we extend investigations started in [3] and construct such potentials and effective dimensions as functions of $r$.

Heavy quarkonium is a system which can probe all scales of QCD. Hence heavy quarkonium presents an ideal laboratory for testing the interplay between perturbative and nonperturbative QCD within a controlled environment. In the last decade, lots of states were found experimentally in the heavy quarkonium mass range that did not fit into the scheme predicted by the constituent quark model, for recent reviews see, for example, reference [4-6]. We hope our method of continual interpolation of the dimension of hadronic space will be useful in that beyond of the standart quarkonium model states.

In Section 2 of this paper, we construct the point charge potential for different dimensions of space. The same problem is considered in Section 3 by using operator fractal calculus. In Section 4 , we describe the quarkonium potential in terms of the potential of a point charge and identify the strong coupling fine structure constant dynamics. Then, in Section 5, we formulate renormdynamics in terms of hamiltonian dynamics and solve corresponding motion equations by numerical and graphical methods, we find corresponding asymptotics. Next, we deal with another example: spaces with compactified dimensions. In Section $6,1 / r^{2}$ potential of a nonlinear extension of the quantum mechanics constructed. Such potentials are ingredients of space compactification problems considered in Section 7. Section 8 deals with Debye screening motivated potentials and its anti-screening counterparts. These potentials are supplemented by a confining factor corresponding to the proper account of the topological effects of the QCD vacuum. The form of the confining potential corresponds to the existence of halo states of nuclear matter. In Section 9, mass parameter effects are motivated and estimated. After conclusions and discussions, several appendices of technical nature and proofs are given.

\section{Coulomb Problem in $D$ Dimensions}

We have the following expression for the solution of the Poisson equation with a point-like source in $D$-dimensional space $[1,7]$ (see Appendix A):

$$
\begin{aligned}
& \Delta \varphi=e \delta^{D}(x) \\
& \varphi(D, r)=-\frac{\Gamma(D / 2)}{2(D-2) \pi^{D / 2}} e r^{2-D}, \\
& V(D, r)=e \varphi(D, r)=-\alpha(D) r^{2-D}, \\
& \alpha(D)=\frac{e^{2} \Gamma(D / 2)}{2(D-2) \pi^{D / 2}}, D \neq 2, r=|x|
\end{aligned}
$$

and, for $D=2$,

$$
\varphi(2, r)=\frac{e}{2 \pi} \ln r, V(2, r)=\frac{e^{2}}{2 \pi} \ln r
$$

In particular, for spatial dimensions 1,3 and 4, we have from (2):

$$
V(1, r)=\frac{e^{2} r}{2}, V(3, r)=-\frac{e^{2}}{4 \pi r}, V(4, r)=-\frac{e^{2}}{4 \pi^{2} r^{2}} .
$$


Now, let us consider Poisson's equation in $D$ dimensions with a general source,

$$
\Delta V(x)=e^{2} \rho(x),
$$

where $V(x)$ is the generalized $D$-dimensional potential, $\rho(x)$ is some source function. We find that for the special case $\rho(x)=\delta(x)$ we obtain the fundamental solution (2) (for textbook derivations using generalized functions, see, e.g., [8]):

$$
E(x)=-\frac{\alpha(D)}{r^{D-2}} .
$$

For a distribution $\rho(x)$ of delta functions over a volume $v$, the corresponding value of $V(x)$ is

$$
V(x)=E * \rho=-\alpha_{D} \int_{v} \frac{\rho(y) d^{D} y}{|x-y|^{D-2}}
$$

where $*$ denotes convolution.

As defined so far, the coupling constant has a mass dimension $d_{e}=(D-3) / 2=-\varepsilon$. To work with a dimensionless coupling constant $e$, we introduce the mass scale $\mu$. Then, the potential energy (2) takes the following form

$$
V(D, r)=-\frac{\Gamma(D / 2)}{2(D-2) \pi^{D / 2}} e^{2} \mu^{2 \varepsilon} r^{2-D}=-\alpha(D)(\mu r)^{2 \varepsilon} / r=-\alpha(D)(x)^{2-D} \mu .
$$

Let us relate the point charge problem to quarkonium and derive the Coulomb coefficient $k=4 / 3 \alpha_{s}$ in the Cornell potential (1). The static potential between two heavy quarks belongs to the fundamental quantities of QCD. In lowest order, in coordinate space (see, e.g., [9])

$$
V_{n}(r)=-C_{n} \frac{\alpha_{s}(\mu)}{r}, C_{n}=C_{n}\left(N_{c}\right), n=1,8,
$$

with $C_{1}\left(N_{c}\right)=C_{F}=\left(N_{c}-N_{c}^{-1}\right) / 2>0$ for the colour-singlet and $C_{8}\left(N_{c}\right)=C_{F}-C_{A} / 2=-1 / 2 N_{c}<0$ for the colour-octet state. $N_{c}$ denotes the number of colors. In QCD, $N_{c}=3$. Thus $C_{1}(3)=(3-1 / 3) / 2=4 / 3$, as we wanted to show.

\section{Fractal Calculus of Quantum Field Theory in Examples}

Let us solve the point charge problem another way-using fractal calculus. The solution in case of a non-integer dimension is, by definition, a fractal (for concise introduction in fractal calculus with some applications see, e.g., [10]). Matrix calculus in QFT perturbation theory [11] can be interpreted as operator fractal calculus. Indeed, with the following definitions

$$
\begin{aligned}
& {\left[\hat{x}_{n}, \hat{p}_{m}\right]=i \delta_{n, m}, \hat{x}_{n}|x\rangle=x_{n}|x\rangle, \hat{p}_{m}|p\rangle=p_{m}|p\rangle,\langle x \mid y\rangle=\delta^{D}(x-y)} \\
& \langle x \mid p\rangle=\frac{1}{(2 \pi)^{D / 2}} \exp (\mathrm{i} p x), \int d^{D} p|p\rangle\left\langle p\left|=\int d^{D} x\right| x\right\rangle\langle x|=1
\end{aligned}
$$

for two point function we have (see Appendix B),

$$
\begin{aligned}
& G(x, y)=\left\langle x\left|\hat{p}^{-2 \alpha}\right| y\right\rangle=A(\alpha)(x-y)^{-2 \beta}, \\
& \beta=\frac{D}{2}-\alpha, A(\alpha)=\frac{\Gamma(\beta)}{2^{2 \alpha} \pi^{D / 2} \Gamma(\alpha)} .
\end{aligned}
$$

As an example, consider the Coulomb potential, the solution of the equation for potential of a point source

$$
\Delta \varphi=e \delta^{D}(x)
$$


Note that, $\Delta=-\hat{p}^{2}$,

$$
\varphi(x)=-e\left\langle 0\left|\frac{1}{\hat{p}^{2}}\right| x\right\rangle=-e \frac{\Gamma(D / 2-1)}{4 \pi^{D / 2}} \frac{1}{|x|^{D-2}}, D \neq 2 .
$$

Using $\Gamma(D / 2-1)=\Gamma(D / 2) /(D / 2-1)$, Formula (13) is easily reduced to (2). As a side note, let us mention another application of fractal calculus-we take the following integral, for composition of two point functions, useful for diagram calculations in Quantum field theories (QFT),

$$
\begin{aligned}
G_{3}(x, y)= & \int d^{D} z(x-z)^{-2 \alpha}(z-y)^{-2 \gamma} \\
= & A^{-1}(\beta) A^{-1}(\delta) \int d^{D} z\left\langle x\left|\hat{p}^{-2 \beta}\right| z\right\rangle\left\langle z|| \hat{p}^{-2 \delta} \mid y\right\rangle \\
= & \frac{A(\beta+\delta)}{A(\beta) A(\delta)}(x-y)^{-2 \eta} \\
& \beta=\frac{D}{2}-\alpha, \delta=\frac{D}{2}-\gamma, \eta=\frac{D}{2}-(\beta+\delta)=\alpha+\gamma-\frac{D}{2}
\end{aligned}
$$

The constructions of this section can be translated in terms of the theory of generalized functions [8]. This, as well as the reconstruction of QFT, will be done elsewhere.

\section{Dimension Dynamics from Cornell Potential}

In this section, we obtain the dynamics of the dimension of hadron space and of the coupling constant as a function of distance between quarks.

Let us compare the Cornell potential and the Coulomb potential with dynamically changing space dimension. Then, we define the dimension $D$ of space from the equality of (1) and (8)

$$
\frac{k-x^{2}}{x^{3-D}}=\alpha(D)=\frac{e^{2} \Gamma(D / 2)}{2(D-2) \pi^{D / 2}}=\alpha_{s} \frac{2 \Gamma(D / 2)}{(D-2) \pi^{(D-2) / 2}}, \alpha_{s}=\frac{e^{2}}{4 \pi} .
$$

For any values of $x$ and $D$, the coupling constant $\alpha_{s}$ thus becomes:

$$
\alpha_{s}(D, x)=\frac{\pi^{(D-2) / 2}}{2 \Gamma(D / 2)}(D-2) \alpha, \alpha=\frac{k-x^{2}}{x^{3-D}}=\left(k-x^{2}\right) x^{D-3} .
$$

At the distinguished values $D=1$ and $D=3$ we have:

$$
\begin{aligned}
& \alpha_{s}(1, x)=\frac{1}{2 \pi}\left(1-\frac{k}{x^{2}}\right), x^{2}>x_{0}^{2}=k \\
& \alpha_{s}(3, x)=k-x^{2}=\alpha_{s}=\frac{e^{2}}{4 \pi}, x^{2}<x_{0}^{2}=k
\end{aligned}
$$

where the inequalities represent the requirement for the coupling constant $\alpha_{s}$ to be positive, see Figure 1.

According to (17) and Figure 1, the hadronic space dimension at point $x=\sqrt{k}=0.72$ changes from 3 to 1 . We may interpret this as a phase transition from quark-gluon (or gluquar) phase to confining (hadron) phase at high temperatures and/or densities of hadronic matter corresponding to distances of order $r=x_{0} a=0.72 \times 2.34 \mathrm{GeV}^{-1}=0.34 \mathrm{fm}$.

In the following section, we will formulate the hamiltonian form of renormdynamics and apply it in the analysis of space dimension and coupling constant dynamics (16). 


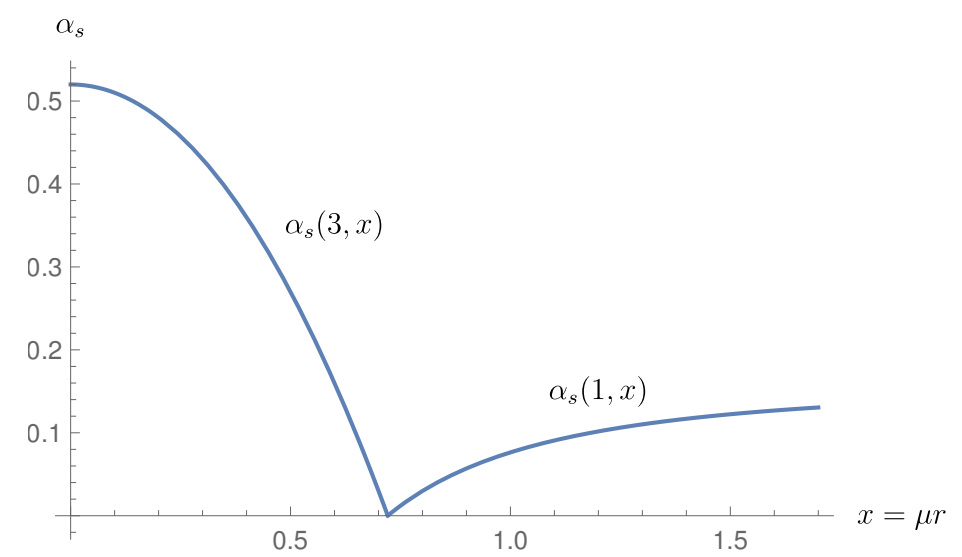

Figure 1. $\alpha_{s}$ as a function of $x=\mu r \in(0,1.7)$ for distinguished values $D=1$ and $D=3$.

\section{Hamiltonian Formulation of the Space Dimension Dynamics}

Here, we will apply the general framework of Hamiltonian mechanics to the equations of renormdynamics. By this method we made good motivation for an explicit form of $D(x)$ : we use a simple example of Hamiltonian mechanics and show how to use it to find the dimension $D$ of space as a function of distance $x$.

Let us consider the simplest Hamiltonian dynamics

$$
\dot{x}_{1}=\left\{x_{1}, H\right\}, \quad \dot{x}_{2}=\left\{x_{2}, H\right\}
$$

for dynamical variables (phase space) $\left(x_{1}, x_{2}\right)$, Hamiltonian $H$

$$
H=\frac{p^{2}}{2 m}+V(x)=\frac{x_{1}^{2}}{2 m}+V\left(x_{2}\right)
$$

and Poisson structure

$$
\{A, B\}=f_{n m} \frac{\partial A}{\partial x_{n}} \frac{\partial B}{\partial x_{m}}=f_{12}\left(\frac{\partial A}{\partial x_{1}} \frac{\partial B}{\partial x_{2}}-\frac{\partial A}{\partial x_{2}} \frac{\partial B}{\partial x_{1}}\right) .
$$

Instead of solving the system of motion equations, having one integral of motion-Hamiltonian, we may find $x_{1}$ from the Hamiltonian, insert it in the motion equation for $x_{2}$ and solve it. Similarly, we consider our coupling constant dynamics. The variables $x, D$ and $\alpha$ are nonnegative, so it is natural to introduce, free from this restriction, variables: $t=\ln x, x_{1}=\ln \alpha_{s}$ and $x_{2}=\ln D$. Then from (15) we obtain the following Hamiltonian and motion equations

$$
\begin{aligned}
& H\left(x_{1}, x_{2}, t\right)=x_{1}-V\left(x_{2}, t\right)=E \Rightarrow x_{1}=V\left(x_{2}, t\right)+E \\
& \dot{x}_{1}=f_{12} \frac{\partial V}{\partial x_{2}} \\
& \dot{x}_{2}=-f_{12}, V\left(x_{2}, t\right)=\ln \left(\frac{\pi^{(D-2) / 2}}{2 \Gamma(D / 2)}(D-2) \frac{k-x^{2}}{x^{3-D}}\right)-E .
\end{aligned}
$$

We may also take $x_{1}=\alpha_{s}=\alpha, E=0$, then

$$
\begin{aligned}
& x_{1}=V\left(x_{2}, t\right)=\left(k-x^{2}\right) x^{D-3}=\left(k-x^{2}\right) x^{\exp \left(x_{2}\right)-3}=\left(k-e^{2 t}\right) e^{t\left(e^{-t}-3\right)}, \\
& \dot{x}_{1}=\frac{\partial V}{\partial x_{2}}=\left(k-x^{2}\right) x^{x^{x_{2}}-3} \ln x e^{x_{2}}=\left(k-e^{2 t}\right) t e^{t\left(e^{-t}-3\right)} e^{-t}, f_{12}=1, \\
& \dot{\alpha}=\beta=t e^{-t} \alpha=\beta_{1} \alpha, \beta_{1}=\ln \frac{\alpha e^{3 t}}{k-e^{2 t}} \\
& \dot{x}_{2}=-1 \Rightarrow x_{2}=-t+\ln c, D=c / x, c=1,
\end{aligned}
$$


Let us return to the general expression (16)

$$
\begin{aligned}
& \alpha_{s}(D, x)=\frac{\pi^{(D-2) / 2}}{2 \Gamma(D / 2)}(D-2) \frac{k-x^{2}}{x^{3-D}}=\frac{\pi^{(1 / x-2) / 2}}{2 \Gamma(1 / 2 x)}(1 / x-2) \frac{k-x^{2}}{x^{3-1 / x}} \\
& =\frac{\pi^{(1 / x-2) / 2}}{2 \Gamma(1 / 2 x)}(1 / x-2)(\sqrt{k}-x) \frac{\sqrt{k}+x}{x^{3-1 / x}}
\end{aligned}
$$

where for simplicity we set the integration constant $c=1$ and defined $D(x)=1 / x$. Note that $x>0$ and $\alpha_{s} \geq 0$ when $x<\min (1 / 2, \sqrt{k})=1 / 2$ or $x>\max (1 / 2, \sqrt{k})=\sqrt{k}=0.72$ while $\alpha_{s}<0$ for $1 / 2<x<0.72$, cf. Figure 2 .

We may exclude the negative interval $(1 / 2,0.72)$ by using different mass scales $\mu$ : $r \mu_{1}=1 / 2, r \mu_{2}=0.72, \mu_{2} / \mu_{1}=1.44$. Alternatively, we may close the negative interval by taking $\sqrt{k}=1 / 2 \Rightarrow \alpha_{s}=3 / 16=0.1875$ in the last formula of (23), c.f. Figure 2 :

$$
\begin{aligned}
& \alpha_{S}(D, x)=\frac{\pi^{(D-2) / 2}}{2 \Gamma(D / 2)}(D-2) \frac{k-x^{2}}{x^{3-D}}=\frac{\pi^{(1 / x-2) / 2}}{2 \Gamma(1 / 2 x)}(1 / x-2) \frac{k-x^{2}}{x^{3-1 / x}} \\
& =\frac{\pi^{1 / 2 x-1}}{\Gamma(1 / 2 x)}(x-1 / 2)^{2} \frac{x+1 / 2}{x^{4-1 / x}} \rightarrow \frac{1}{2 \pi x^{2}}, x \gg 1
\end{aligned}
$$

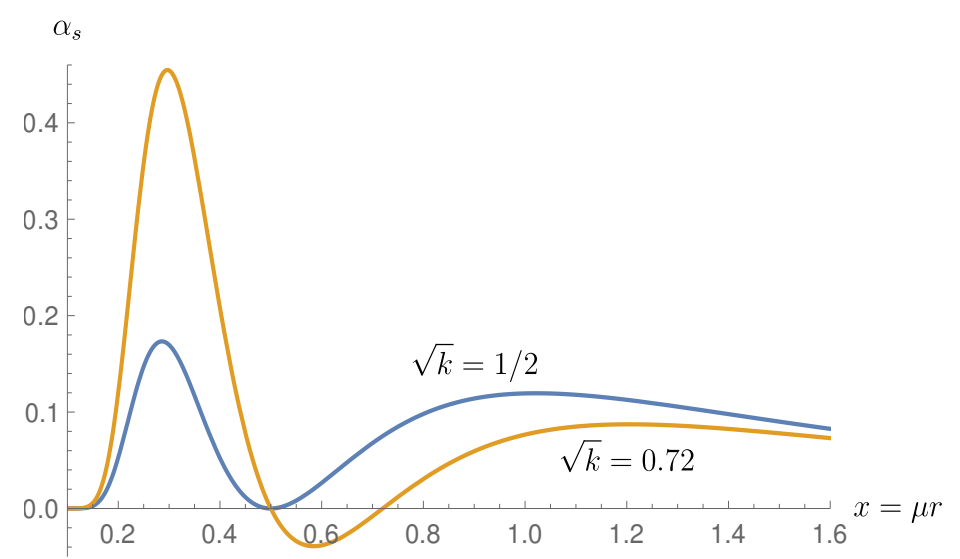

Figure 2. $\alpha_{s}$ as a function of $x=\mu r \in(0.1,1.6)$ for $\sqrt{k}=0.72$ with the negative interval and with $\sqrt{k}=1 / 2 \Rightarrow \alpha_{s}=3 / 16=0.1875$, with the negative interval closed.

If we want for the dimension usual asymptotic behaviour for Cornell potential: $D(\infty)=1, D(0)=3$, we may take, e.g., the following solution

$$
\begin{aligned}
& D(x)=3-2 \tanh (c x), \dot{D}=-\frac{2 c}{\cosh ^{2}(c x)}=\beta(D), \\
& \beta(D)=-c(D-1)(5-D) / 2 .
\end{aligned}
$$

Now, from the motion equation for $x_{1}=\alpha_{s}$, and the new structure function

$$
\begin{aligned}
& \dot{x}_{2}=-f_{12} \Downarrow \\
& f_{12}=-c(D-5)(D-1) /(2 D),
\end{aligned}
$$

we construct $\beta$-function given in parametric form

$$
\begin{aligned}
& \dot{x}_{1}=\beta=f_{12} \frac{\partial V}{\partial x_{2}} \\
& \alpha_{s}(D, x)=\frac{\pi^{(D-2) / 2}}{2 \Gamma(D / 2)}(D-2) \frac{k-x^{2}}{x^{3-D}}
\end{aligned}
$$




$$
\dot{\alpha}_{s}=\frac{\partial \alpha_{s}}{\partial x}+\frac{\partial \alpha_{s}}{\partial D} \dot{D}
$$

We close the negative interval for $\alpha_{s}$, see Figure 3, defining the parameter $c$ as

$$
D=2=3-2 \tanh (c \sqrt{k}) \Rightarrow \tanh (c \sqrt{k})=\frac{1}{2}, \sqrt{k}=0.72, c=0.76 .
$$

For ultraviolet value we have

$$
\alpha_{s}(D=3, x=0)=\pi^{1 / 2} k / 2 / \Gamma(3 / 2)=k=0.52 .
$$

For infrared value we have

$$
\alpha_{S}(D=1, x=\infty)=\pi^{-1 / 2}(-1)(-1) / 2 / \Gamma(1 / 2)=\frac{1}{2 \pi} \simeq 0.16 .
$$

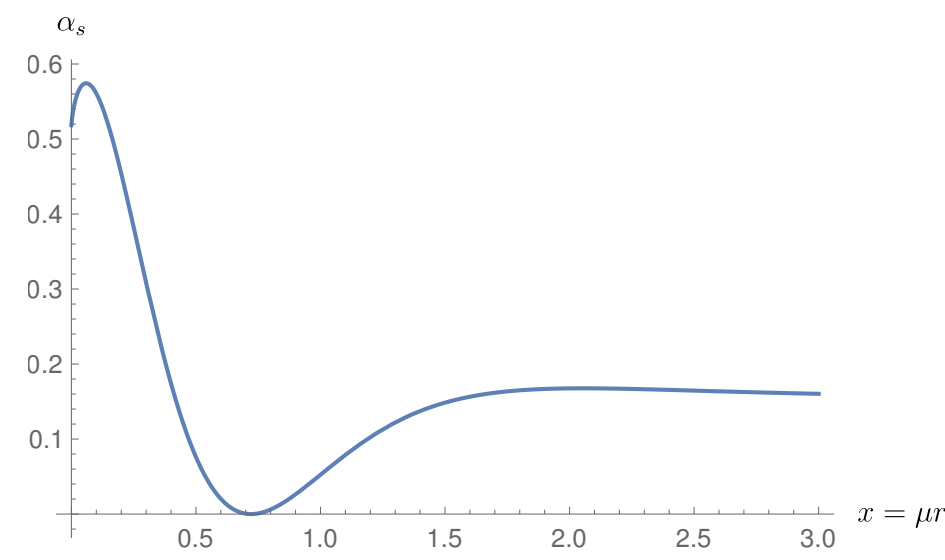

Figure 3. $\alpha_{s}$ as a function of $x=\mu r \in(0,3)$ when $D(0)=3, D(\infty)=1$.

\section{Extended Quantum Mechanics and Conformal Potential}

In this section, we derive $1 / r^{2}$ type potentials in extended quantum mechanics [12]. These potentials serve as a basis to the following section where we consider potentials in spaces where some coordinates are compactified.

In extended quantum mechanics, the usual Schroedinger equation was completed by corresponding motion equation for potential $V$ in the following Hamiltonian system

$$
\begin{aligned}
& i \psi_{t}=-\Delta \psi+V \psi, \\
& i V_{t}=\Delta V-\frac{1}{2} V^{2},
\end{aligned}
$$

with a partial solution for the potential

$$
V=\frac{4(4-D)}{r^{2}}
$$

For dimension of space $D>4$, we have attractive potential with the possibility of quantum-mechanical bound states. We suppose that at small scales we have $D$-dimensional Euclidean (or Riemannian) space with $1 / r^{2}$ potential. At usual scales we observe three dimensional space, so extra $D-3$ dimensions are compactified, e.g., as $D-3$ dimensional torus $\mathbb{T}^{D-3}=\left(\mathbb{T}^{1}\right)^{D-3}$. In the minimal case, $D=5$ and we need a two dimensional torus. The extra dimension may be compactified also in $S^{2}$ sphere $\left(S^{D-3}\right.$ sphere). Note that the circle $\mathbb{T}^{1}$ is $S^{1}$ sphere. 
In a more general case of the potential motion equation,

$$
\begin{aligned}
& i \psi_{t}=-\Delta \psi+\bar{V} \psi, \\
& i V_{t}=\Delta V-\frac{1}{n} V^{n}, \bar{V}=V^{n-1}
\end{aligned}
$$

we have the following solution (see Appendix C).

$$
V=\left(\frac{2 n}{n-1}\left(\frac{2 n}{n-1}-D\right) \frac{1}{r^{2}}\right)^{\frac{1}{n-1}}, \bar{V}=V^{n-1}=\frac{2 n}{n-1}\left(\frac{2 n}{n-1}-D\right) \frac{1}{r^{2}} .
$$

\section{Compactification and Dimension Dynamics}

In this section we use the idea of interpolating (fractal) space dimension in another class of models: with compactified dimensions. We implement the idea in the simplest case of one compact dimension and then give some general arguments for cases with several compact dimensions.

Let us take one of the dimensions $y$ as circle with radius $R$. This corresponds to the periodic structure with a point charge sources at each point $y_{n}=y+2 \pi R n, n=0, \pm 1, \pm 2, \ldots$

$$
\begin{aligned}
& \Delta \varphi=e \sum_{n} \delta^{D}(x) \delta\left(y_{n}\right), \varphi(D, r, y)=\sum_{n} \varphi\left(D, r, y_{n}\right), \\
& V(D, r, y)=-\alpha(D+1) \sum_{n=-\infty}^{\infty}\left(r^{2}+(2 \pi R n+y)^{2}\right)^{(1-D) / 2} .
\end{aligned}
$$

When $D=3$, the potential (35) can be writen in a closed form [1]

$$
V_{3}(r, y)=-\frac{\alpha(4)}{2 R r} \frac{\sinh (r / R)}{\cosh (r / R)-\cos (y / R)}= \begin{cases}\alpha(4) /(2 R r), & r \gg R \\ \alpha(4) /\left(r^{2}+y^{2}\right), & r, y \ll R\end{cases}
$$

where $\alpha(4) /(2 R)=\alpha(3)$. Alternatively, we can rewrite (36) as

$$
V_{3}(r, y)=-\frac{\alpha(4)}{4 R r}\left[\operatorname{coth}\left(\frac{r+\mathrm{i} y}{2 R}\right)+\operatorname{coth}\left(\frac{r-\mathrm{i} y}{2 R}\right)\right],
$$

or, using

$$
A^{-\alpha}=1 / \Gamma(\alpha) \int_{0}^{\infty} d t t^{\alpha-1} e^{-t A},
$$

by means of the Theta function as

$$
V_{3}(r, y)=-\alpha(4) \int_{0}^{\infty} d t e^{-t r^{2}} \sum_{-\infty}^{\infty} e^{-t(2 \pi R n+y)^{2}}=-\alpha(4) \int_{0}^{\infty} d t e^{-t r^{2}} \frac{\theta\left(\frac{\mathrm{i} y}{2 \pi R}, \exp \left(\frac{\mathrm{i}}{4 R^{2} t}\right)\right)}{2 R \sqrt{\pi} \sqrt{t}},
$$

where $\theta(z, \tau)=$ is given in Appendix D.

Dimension Dynamics with One Compact Dimention

For $y=0$, the potential takes the following simple form

$$
V_{3}(r, y=0)=-\frac{\alpha(3)}{r} \operatorname{coth} \frac{r}{2 R} .
$$

For $y=\pi R$, we have

$$
V_{3}(r, y=\pi R)=-\frac{\alpha(3)}{r} \tanh \frac{r}{2 R}
$$


From (36), we see that for big $r$, the effective dimension of space is 3 and for small $r$ is 4 . For intermediate scales, the effective dimension might change smoothly from 3 to 4 . Integrating by coordinate $y$ (or angle $\vartheta$, see Appendix E) we define mean potential depending only on the variable $r$ [1],

$$
\bar{V}_{3}(r)=\frac{1}{2 \pi} \int_{0}^{2 \pi} d \vartheta V_{3}(r, \vartheta)=-\frac{\alpha_{3}}{r}, \quad \alpha_{3} \equiv \alpha(3) .
$$

As in the Cornell potential case (cf. Equality (15)), we define the dimension dynamics from equality between the Coulomb potentials (8) and (36)

$$
\begin{aligned}
& \frac{\alpha(4)}{2 r} \frac{\sinh (r / R)}{\cosh (r / R)-\cos (y / R)}=\alpha(D)(x)^{2-D}, \alpha(4)=2 R \alpha(3) \\
& \mu=1 / R, x=\mu r,
\end{aligned}
$$

We may take some interpolating dimension, e.g.,

$$
D(x)=4-\tanh x,
$$

and define the coupling function dynamics as

$$
\begin{aligned}
& \alpha(x, \varphi)=\alpha_{3} x^{D-3} \frac{\sinh x}{\cosh x-\cos \varphi}, x=r / R, \varphi=y / R \in[0,2 \pi), \\
& \alpha(x, 0)=\alpha_{3} x^{D-3} \operatorname{coth}(x / 2)=\left\{\begin{array}{cc}
2 \alpha_{3} & x \ll 1 \\
\alpha_{3} & x \gg 1
\end{array},\right. \\
& \alpha(x, \pi)=\alpha_{3} x^{D-3} \tanh (x / 2)=\left\{\begin{array}{cc}
\alpha_{3} x^{2} / 2 & x \ll 1 \\
\alpha_{3} & x \gg 1
\end{array} .\right.
\end{aligned}
$$

The last case corresponds to the asymptotic freedom at small $r / R$ scales, see Figure 4 . From the point of view of the compact subspace, it corresponds to infrared asymptotic freedom.

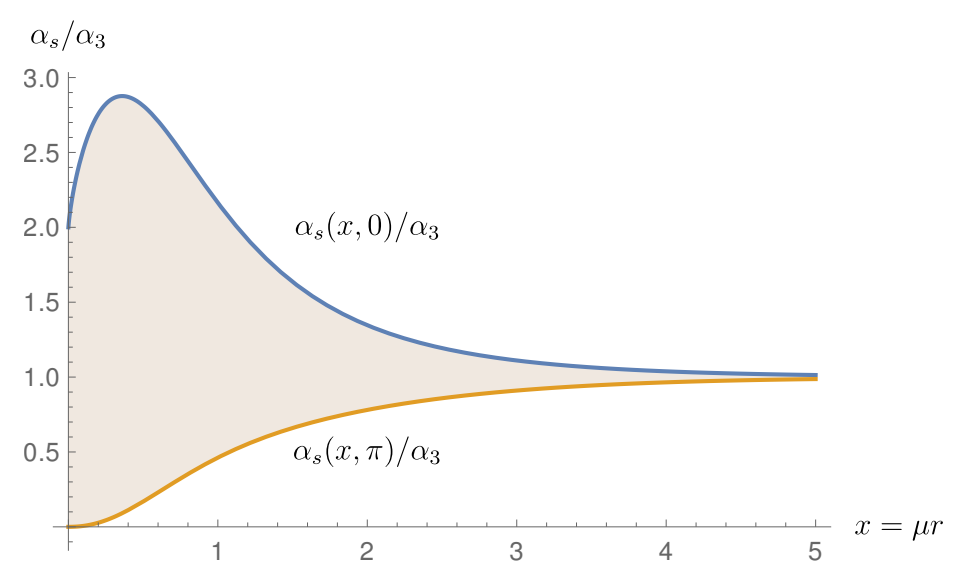

Figure 4. $\alpha_{s}(x, 0) / \alpha_{3}$ and $\alpha_{s}(x, \pi) / \alpha_{3}$ as a function of $x=\mu r \in(0.1,5)$.

The global maximum value is $\alpha_{s}(x, \varphi) / \alpha_{3}=2.87644$ at $\varphi=0, x_{c}=0.360028, D\left(x_{c}\right)=3.65476$, see Figure 5. So, we have ultraviolet and infrared fixed points and intermediate fixed point of renormdynamics. Beta functions for the normed coupling constant: $a=\alpha_{s}(x, \varphi) / \alpha_{3}$ are

$$
\begin{aligned}
& a_{\varphi}=\frac{\partial a}{\partial \varphi}=-\frac{\sin \varphi}{\cosh x-\cos \varphi} a, \\
& a_{x}=x \frac{\partial a}{\partial x}=\left(x D_{x} \ln x+D-3+x\left(\operatorname{coth} x-\frac{\sinh x}{\cosh x-\cos \varphi}\right)\right) a,
\end{aligned}
$$




$$
D=4-\tanh x, D_{x}=-1 / \cosh ^{2} x .
$$

From the first equation of the system we see that $a_{\varphi}=0$ for $a \neq 0$ at $\varphi=0, \pi$. At $\varphi=0$, we have maximum. At $\varphi=\pi$, we have infrared fixed point, see Figure 6 .

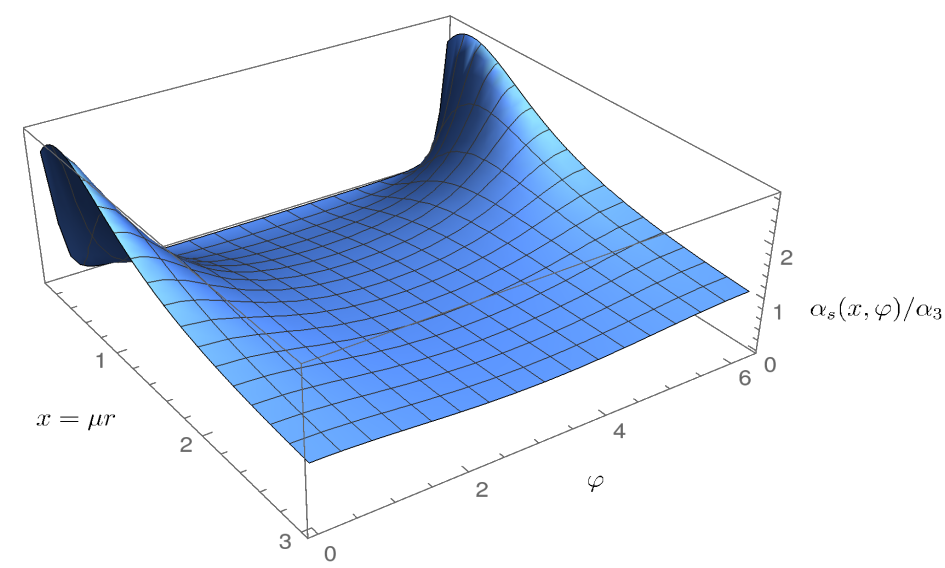

Figure 5. $\alpha_{s}(x, \varphi) / \alpha_{3}$ as a function of $x=\mu r \in(0.1,3), \varphi \in(0,2 \pi)$.

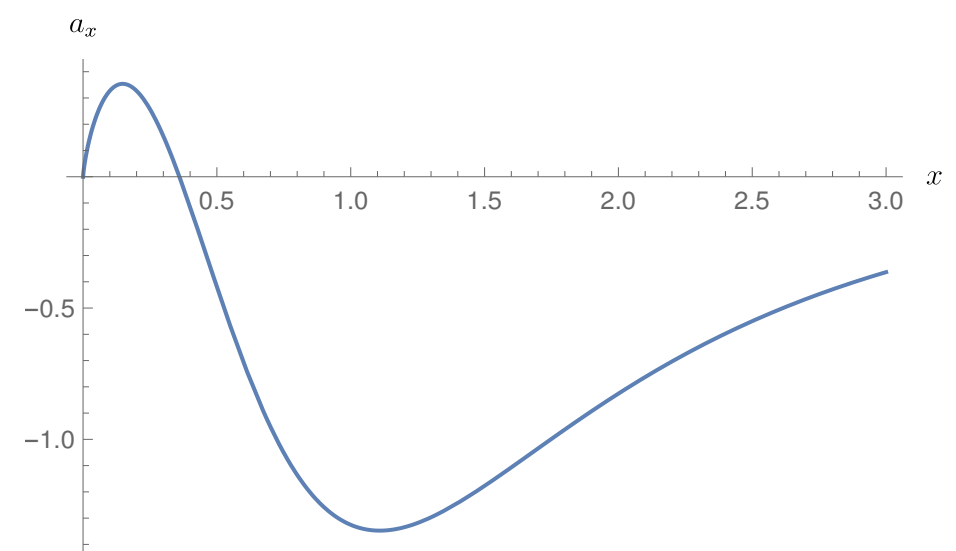

Figure 6. Beta function $a_{x}$, cf. (46), as a function of $x=\mu r \in(0,3)$, with $\varphi=0$. The curve intersects the $x$-axis, determining the global maximum of $\alpha_{s}(x, \varphi) / \alpha_{3}=2.87644$ at $\varphi=0, x_{c}=0.360028$, $D\left(x_{c}\right)=3.65476$.

For a point quark inside hadron of size $R$ at a temperature $T$ we have

$$
\begin{aligned}
& \Delta \varphi=e \sum_{k, l, n, m} \delta\left(\tau_{k}\right) \delta\left(x_{l}\right) \delta\left(y_{n}\right) \delta\left(z_{m}\right), \\
& \varphi(0, \tau, x, y, z)=\sum_{k, l, n, m} \varphi\left(0, \tau_{k}, x_{l}, y_{n}, z_{m}\right), \\
& V(0, \tau, x, y, z)=-\alpha_{4} \sum_{k, l, n, m=-\infty}^{\infty}\left((2 \pi k / T+\tau)^{2}\right. \\
& \left.+\left(2 \pi R_{1} l+x\right)^{2}+\left(2 \pi R_{2} n+y\right)^{2}+\left(2 \pi R_{3} m+z\right)^{2}\right)^{-1} \\
& =-\alpha_{4} \int_{0}^{\infty} d t t B_{0}(t, \tau) B_{1}(t, x) B_{2}(t, y) B_{3}(t, z), \\
& B_{1}(t, x)=\sum_{n=-\infty}^{\infty} e^{-t\left(2 \pi R_{1} n+x\right)^{2}}=e^{-t x^{2}} \theta\left(2 \mathrm{i} R_{1} x t, 4 \pi \mathrm{i} R_{1}^{2} t\right), \ldots, R_{0}=1 / T
\end{aligned}
$$

where we have written the sums by means of the Theta function (see Appendix D). 
For the sake of completeness, let us state the general expression for the potential in space $\mathbb{R}^{D} \times \mathbb{T}^{d}$ where $\mathbb{T}^{d}=S^{1} \times \cdots \times S^{1}$ (d-times) is the $d$-dimensional torus. $D$ refer to the "big" dimensions $\mathbf{x}=\left(x_{1}, \ldots x_{D}\right)$, whereas $d$ to the "small-compactified" ones $\mathbf{y}=\left(y_{1}, \ldots y_{d}\right)$. Then

$$
\begin{aligned}
& \Delta \varphi=e \sum_{n_{1}, \ldots, n_{d}} \delta^{D}(\mathbf{x}) \delta\left(y_{1, n_{1}}\right) \ldots \delta\left(y_{d, n_{d}}\right) \\
& \varphi\left(D, d, r, y_{1}, \ldots, y_{d}\right)=\sum_{n_{1}, \ldots, n_{d}} \varphi\left(D, d, r, y_{1, n_{1}}, \ldots, y_{d, n_{d}}\right) \\
& V_{D, d}\left(r, y_{1}, \ldots, y_{d}\right)=-\alpha_{D+d} \sum_{n_{1}, \ldots, n_{d}=-\infty}^{\infty}\left(r^{2}+\left(2 \pi R_{1} n_{1}+y_{1}\right)^{2}+\cdots+\left(2 \pi R_{d} n_{d}+y_{d}\right)^{2}\right)^{-(D+d-2) / 2} \\
& =-\frac{\alpha_{D+d}}{\Gamma\left[\frac{D+d-2}{2}\right]} \int_{0}^{\infty} d t t^{\frac{D+d-4}{2}} e^{-t r^{2}} \sum_{n_{1}, \ldots, n_{d}=-\infty}^{\infty} e^{-t\left(2 \pi R_{1} n_{1}+y_{1}\right)^{2}} \ldots e^{-t\left(2 \pi R_{d} n_{d}+y_{d}\right)^{2}} \\
& =-\frac{\alpha_{D+d}}{\Gamma\left[\frac{D+d-2}{2}\right]} \int_{0}^{\infty} d t t^{\frac{D+d-4}{2}} e^{-t r^{2}} \prod_{i=1}^{d} B_{i}\left(t, y_{i}\right) \\
& B_{i}\left(t, y_{i}\right)=\sum_{n_{i}=-\infty}^{\infty} e^{-t\left(2 \pi R_{i} n_{i}+y_{i}\right)^{2}}=e^{-t y_{i}^{2}} \theta\left(2 \mathrm{i} R_{i} y_{i} t, 4 \pi \mathrm{i} R_{i}^{2} t\right)
\end{aligned}
$$

where we have again used (38) and written the sums in the expressions for $B_{i}$ by means of the Theta function (see Appendix D).

Note that the B-factors in the integrand $\sim t^{-d / 2}$ for small $t$, so the integral is divergent when $D \leq 2$. For example, for total dimension $D+d=4$ and $d=2$, the integral is divergent. We may regularize the integral by restricting summation by some $N$ or consider analytic continuation $D+d+\epsilon$ in the monomial factor of the integral. We may define the same conditions from direct form of the sum, before integral transform. Divergent part of the sum is estimated by integral

$$
\int d^{d} x x^{2-D-d} \sim \int d x x^{1-D} \sim x^{2-D}
$$

which is divergent for $D<2$. For $D=2$, divergent part of the sum is estimated by integral

$$
\int d^{d} x x^{-d} \sim \int d x / x \sim \ln x
$$

which gives logarithmic divergence. In the world of confining potentials, $D \leq 2$, there are no macroscopic systems of infinite size or infinite number of constituents.

\section{Debye Screening, Modified Gluon Propagator}

It is known that the force between two charges, $e$ and $-e$, changes when the system is placed in a medium. In an ionized plasma, the $1 / r$ potential turns into Yukawa-Debye screening [13] (see also [14])

$$
V(r)=-\frac{\alpha e^{-\mu r}}{r}=-\frac{\alpha}{r}-\sigma r+\ldots, \sigma=\alpha \mu^{2} / 2
$$

where we marked with ellipsis the irrelevant constant term $\alpha \mu$ and terms of order higher than linear in $r$ (irrelevant for small $r$ ). In expanded form it reminds of the "Cornell potential" (1)

$$
V(r)=-\frac{k}{r}+\sigma r, \sigma=1 / a^{2}
$$

but the sign of the string tension $\sigma$ is opposite. The positive sign corresponds to the confined phase, negative sign to screened (deconfined) phase. We can take (test) as a confining potential the following one (cf. Figure 7):

$$
V(r)=-\frac{\alpha \cos \mu r}{r}=-\frac{\mu \alpha \cos (x)}{x}=-\frac{\alpha}{r}+\sigma r+\ldots, \sigma=\alpha \mu^{2} / 2, x=\mu r .
$$




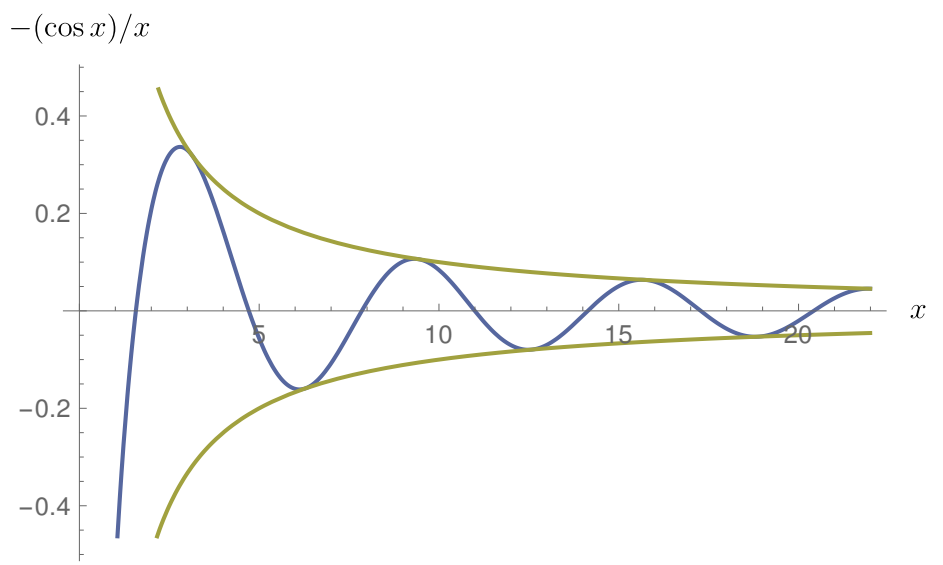

Figure 7. Potential (53).

The confining potential turns into a deconfining one when $\mu^{2}$ changes sign or when exchange particle becomes tachyon.

In paper [15], by proper account of the compact nature of SU(3) gauge group that gives rise to the periodic $\theta$-vacuum of the theory, the gluon propagator was modified as

$$
G(p)=\left(p^{2}+\chi / p^{2}\right)^{-1}=\frac{p^{2}}{p^{4}+\chi}=\frac{1}{2}\left(\frac{1}{p^{2}+i \sqrt{\chi}}+\frac{1}{p^{2}-i \sqrt{\chi}}\right)
$$

which gives the potential (cf. Figure 8):

$$
\begin{aligned}
& V(r)=-\frac{\alpha \cosh \mu r \cos \mu r}{r}=-\frac{\mu \alpha \cosh x \cos x}{x}=\mu \alpha\left(-\frac{1}{x}+\frac{x^{3}}{6}+\ldots\right), \\
& x=\mu r, \mu=\sqrt[4]{\chi} / \sqrt{2}
\end{aligned}
$$

where $\chi$ is the Yang-Mills topological susceptibility related to the $\eta^{\prime}$ mass by the Witten-Veneziano relation,

$$
\chi=\frac{F_{\pi}^{2}}{2 N_{f}}\left(m_{\eta^{\prime}}^{2}+m_{\eta}^{2}-2 m_{K}^{2}\right) \simeq(180 \mathrm{MeV})^{4}, \mu=\sqrt[4]{\chi} / \sqrt{2}=127 \mathrm{MeV} .
$$

The topological susceptibility in this formula is the only quantity which is by definition calculable in gluodynamics. Early papers of its calculation are [16-18], and more recently [19].

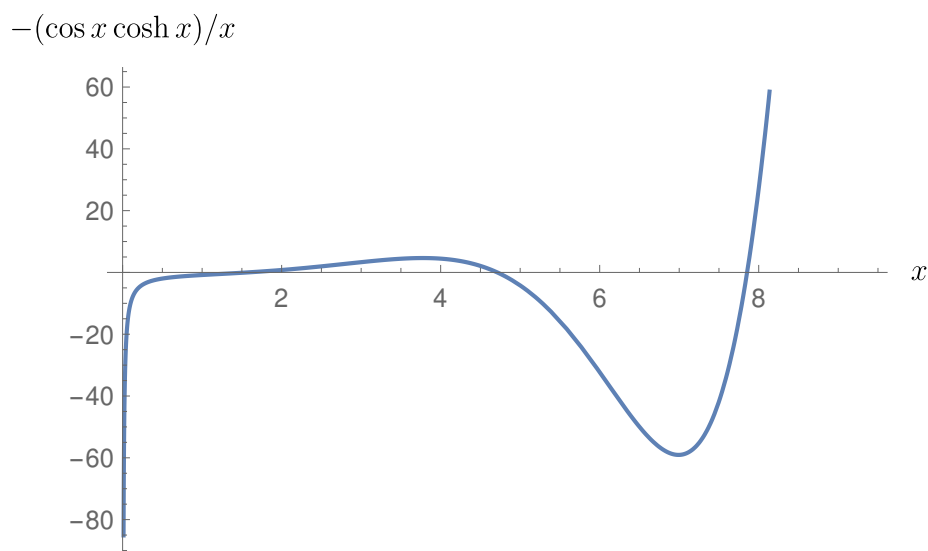

Figure 8. Confining potential (55). 
Potential (55) is well motivated and confining. In the minimum of the potential (55) bound states "bags" have size of the order of $11 \mathrm{fm}$,

$$
r=7 / \mu=7 / 0.127 \mathrm{GeV}^{-1}=11 \mathrm{fm}, \mathrm{GeV}^{-1} \simeq 0.2 \mathrm{fm},
$$

and can give rise to long lived states corresponding to hadronic halos or galactic (in case of gravitational) halos.

\section{Variable Mass and Momentum-Space Formulations of Dimension Dynamics}

We may change the long distance behaviour of the Coulomb potential by including a variable mass term

$$
\begin{aligned}
& \triangle V-m^{2} V=-e^{2} \delta^{D}(x), \delta^{D}(x)=(2 \pi)^{-D} \int d^{D} p e^{\mathrm{i} p x} \Downarrow \\
& V(r)=\frac{e^{2}}{(2 \pi)^{D}} \int d^{D} p\left(p^{2}+m^{2}\right)^{-1} e^{\mathrm{i} p x} \sim r^{2-D} e^{-m r} \sim r^{2-d}, \\
& m \Rightarrow m(r)=a \ln (\mu r) / r, d=D+a .
\end{aligned}
$$

Note that the $m^{2}$ term plays the role of a potential. Comparing this term with Expression (32), we find the corresponding dimension dynamics: $D(r)=4-a^{2} \ln ^{2} \mu r / 4$, from which we may estimate parton size $r_{0}$, from hadron size $R \sim 1 \mathrm{fm}$ and condition $D(r)=0: r_{0}=R \exp (-4 / a)$. The parton size $r_{0} \leq 0.3 \mathrm{fm}$-valence quark size, so $a \leq 4$.

In momentum representation, explicit dependence on spatial dimension is contained in the volume form. Form-factor may cause the dimension to depend on momentum. We may construct form-factors corresponding to transition from space-time dimension 4 on small scales, to dimension 2 and then 1 (only a time dimension remains-which is equivalent to confinement) at large scales.

\section{Discussion and Summary}

The main idea of this paper is to change the dimension of the hadronic space continually with the distance between valence quarks and using advanced methods of the fractal calculus obtain qualitatively new results for the description of the quarkonium potential. The most important bonus we received is the dynamics of the coupling constant as a function of distance (and angle, in the case of a compactified extra dimension).

We considered spherically symmetric solutions of the Poisson equation for a point charge. For these solutions, the Laplace operator in radial variables permits an analytic continuation for non-integral values of spatial dimensions. We assign such geometric characteristics to quarkonium, considered to be a hadronic state in a fractal space whose dimensionality changes continually with distance. More precisely, as the distance between valence quarks rises, the dimension of space of hadronic matter changes continually from three to one.

The same idea was realized also in the case of one compact dimension. In unified field theories, extra dimensions are compactified. On small scales, the space dimension is an integer number, and at large scales another one, smaller than the former one. In our approach we interpolate between this two dimensions smoothly, passing fractal geometries and using fractal calculus methods.

The results of this theoretical work could be used for possible improvements in the description of quarkonium spectra and for space (de)compactification problems in the unified field and extended particle models.

Author Contributions: Conceptualization, N.M.; methodology, M.B. and N.M.; software, M.B. and N.M.; validation, M.B. and N.M.; formal analysis, M.B. and N.M.; investigation, M.B. and N.M.; resources, N.M.; data curation, M.B. and N.M.; writing-original draft preparation, M.B. and N.M.; writing-review and editing, M.B. and N.M.; visualization, M.B. and N.M.; supervision, M.B. and N.M.; project administration, M.B. and N.M.; funding acquisition, M.B. and N.M. All authors have read and agreed to the published version of the manuscript. 
Funding: The first author was supported from EU Regional Development Fund-Project No. CZ.02.1.01/0.0/0.0/16_019/0000766.

Conflicts of Interest: The authors declare no conflict of interest. The funders had no role in the design of the study; in the collection, analyses, or interpretation of data; in the writing of the manuscript, or in the decision to publish the results.

\section{Appendix A. Fundamental Solution of the Point Charge Problem}

$$
\begin{aligned}
\int d^{D} x \Delta \varphi & =\Omega_{D} r^{D-1} \frac{d}{d r} \frac{a_{D}}{r^{D-2}}=-(D-2) \Omega_{D} a_{D}=e, a_{D}=-\frac{e}{(D-2) \Omega_{D}}, a_{3}=-\frac{e}{4 \pi}, \\
\int d^{2} x \Delta \varphi & =\Omega_{2} r \frac{d}{d r}\left(a_{2} \ln r\right)=2 \pi a_{2}=e, a_{2}=\frac{e}{2 \pi} \\
\int d x^{D} e^{-x^{2}} & =\left(2 \pi \int_{0}^{\infty} d r r e^{-r^{2}}\right)^{D / 2}=\pi^{D / 2}=\Omega_{D} \int_{0}^{\infty} d r r^{D-1} e^{-r^{2}} \\
= & \frac{\Omega_{D}}{2} \Gamma(D / 2), \Omega_{D}=\frac{2 \pi^{D / 2}}{\Gamma(D / 2)} .
\end{aligned}
$$

\section{Appendix B. Derivation of the Main Formula of the Operator Fractal Calculus}

$$
\begin{aligned}
G(x, y) & =\frac{1}{\Gamma(\alpha)} \int_{0}^{\infty} d t t^{\alpha-1}\left\langle x\left|e^{-t \hat{p}^{2}}\right| y\right\rangle \\
& =\frac{1}{\Gamma(\alpha)} \int_{0}^{\infty} d t t^{\alpha-1} \int d^{D} p\langle x \mid p\rangle\langle p \mid y\rangle \exp \left(-t p^{2}\right) \\
& =\frac{\Gamma\left(\frac{D}{2}-\alpha\right)}{\Gamma(\alpha) 2^{2 \alpha} \pi^{D / 2}}(x-y)^{-2(D / 2-\alpha)}
\end{aligned}
$$

In coordinate representation, $\hat{p}_{n}=-\mathrm{i} \partial / \partial x_{n}$, we have D-dimensional fractal calculus.

\section{Appendix C. Extended Quantum Potential}

Let us consider the Ansatz $V=A r^{m}$,

$$
\begin{aligned}
& (m(m-1)+(D-1) m) r^{m-2}-\frac{1}{n} r^{n m} A^{n-1}=0 \Downarrow \\
& m-2=n m \Rightarrow m=\frac{2}{1-n} ; \\
& m(m+D-2)-\frac{1}{n} A^{n-1}=0 \Rightarrow A=(m n(D+m-2))^{1 /(n-1)}=\left(\frac{2 n}{n-1}\left(\frac{2 n}{n-1}-D\right)\right)^{1 /(n-1)}, \\
& \bar{V}=V^{n-1}=\frac{2 n}{n-1}\left(\frac{2 n}{n-1}-D\right) \frac{1}{r^{2}}, \\
& D \equiv D_{n}=\frac{2 n}{n-1}, D_{2}=4, D_{3}=3, D_{-1}=1, D_{0}=0 .
\end{aligned}
$$

\section{Appendix D. Theta Functions}

Theta functions [20] are the analytic functions $\theta(z, \tau)$ in 2 variables defined by

$$
\theta(z, \tau)=\sum_{n \in \mathbb{Z}} \exp \left[\mathrm{i} \pi\left(\tau n^{2}+2 n z\right)\right]=1+2 \sum_{n \geq 1} \exp \left(\mathrm{i} \pi \tau n^{2}\right) \cos (2 \pi n z),
$$

where $z \in \mathbb{C}$ and $\tau \in \mathbb{H}$, the upper half plane $\operatorname{Im} \tau>0$. The series converges absolutely and uniformly on compact sets. 


\section{Appendix E. Integrals}

$$
\begin{gathered}
\int_{0}^{2 \pi} \frac{d \vartheta}{a+b \cos \vartheta}=\frac{2 \pi}{\sqrt{a^{2}-b^{2}}}=\frac{2 \pi}{\left|c^{2}-1\right|}, a=c^{2}+1, b=2 c \\
\int_{0}^{2 \pi} d \vartheta \frac{e^{i \vartheta}}{a+b \cos \vartheta}=\int_{0}^{2 \pi} d \vartheta \frac{\cos \vartheta}{a+b \cos \vartheta}=\frac{-b}{a+\sqrt{a^{2}-b^{2}}} \frac{2 \pi}{\sqrt{a^{2}-b^{2}}}
\end{gathered}
$$

Let us take the following integral

$$
I(a)=\int_{0}^{\pi} \frac{d \vartheta}{a^{2}+1-2 a \cos \vartheta}=\frac{\pi}{\left|a^{2}-1\right|}=\left\{\begin{array}{cc}
\pi / a^{2}, & a^{2} \gg 1 \\
\pi, & a^{2} \ll 1
\end{array} .\right.
$$

Obviously, $I(1)=\infty$, but

$$
\begin{aligned}
& I(1)=\frac{1}{2} \int_{0}^{\pi} \frac{d \vartheta}{1-\cos \vartheta}=\frac{1}{4} \int_{0}^{\pi} \frac{d \vartheta}{\sin ^{2} \frac{\vartheta}{2}}=\frac{1}{2} \int_{0}^{1} \frac{d x}{\left(1-x^{2}\right)^{3 / 2}} \\
& =\frac{1}{4} \int_{0}^{1} \frac{d y}{y^{1 / 2}(1-y)^{3 / 2}}=B(1 / 2,-1 / 2)=\frac{1}{4} \frac{\Gamma(1 / 2) \Gamma(-1 / 2)}{\Gamma(0)}=0, ? ! \\
& B(\alpha, \beta)=\int_{0}^{1} d x x^{\alpha-1}(1-x)^{\beta-1}=\frac{\Gamma(\alpha) \Gamma(\beta)}{\Gamma(\alpha+\beta)}, \text { Real } \alpha, \beta>0 .
\end{aligned}
$$

In our case $a=\exp (r / R)>1$. Let us take corresponding integral,

$$
\begin{aligned}
& I=\frac{1}{a} \int \frac{d \vartheta}{b+2 \cos \vartheta}=\frac{1}{\mathrm{i} a} \int \frac{d z}{z^{2}+b z+1} \\
& =I(z, a)=\frac{1}{\mathrm{i} a(a-1 / a)} \ln \frac{z+a}{z+1 / a}, \\
& I(a)=I(-1, a)-I(1, a)=\frac{1}{\mathrm{i} a(a-1 / a)} \ln \frac{(-1+a)(1+1 / a)}{(-1+1 / a)(1+a)}=\frac{\pi}{a^{2}-1}, \\
& b=a+1 / a, z=e^{\mathrm{i} \vartheta} .
\end{aligned}
$$

We may calculate the same integral by residue formula

$$
\begin{aligned}
& \oint \frac{d z}{(z+a)(z+1 / a)}=2 \pi \mathrm{i} \begin{cases}1 /(-a+1 / a), & |a|<1 \\
1 /(-1 / a+a), & |a|>1 .\end{cases} \\
& I(a)=\frac{1}{\mathrm{i} a} 2 \pi \mathrm{i}\left\{\begin{array}{ll}
1 /(-a+1 / a), & |a|<1 \\
1 /(-1 / a+a), & |a|>1 .
\end{array}\right\}=\frac{2 \pi}{\left|a^{2}-1\right|} .
\end{aligned}
$$

Now,

$$
\begin{aligned}
& I=\int_{0}^{2 \pi} \frac{d \vartheta}{a^{2}+1-2 a \cos \vartheta}=I(a)+I(-a)=\frac{2 \pi}{\left|a^{2}-1\right|}=\frac{\pi \exp (-r / R)}{\sinh (r / R)}, \\
& \frac{1}{2 \pi} \int_{0}^{2 \pi} \frac{d \vartheta}{\cosh (r / R)-\cos \vartheta}=\frac{2 a}{a^{2}-1}=\frac{1}{\sinh (r / R)}, a=\exp (r / R) .
\end{aligned}
$$




\section{References}

1. Bureš, M.; Siegl, P. Hydrogen atom in space with a compactified extra dimension and potential defined by Gauss law. Ann. Phys. 2015, 354, 316-327. [CrossRef]

2. Eichten, E.; Gottfried, K.; Kinoshita, T.; Lane, K.D.; Tung-Mow, Y. Charmonium: The Model. Phys. Rev. D 1978, 10, 3090. [Erratum: Phys. Rev. 1980, D21, 313.]

3. Bureš, M.; Makhaldiani, N. Space Dimension Dynamics and Modified Coulomb Potential of Quarks-Dubna Potentials. Phys. Part. Nucl. Lett. 2019, 16, 620-624. [CrossRef]

4. Esposito, A.; Pilloni, A.; Polosa, A.D. Multiquark resonances. Phys. Rept. 2016, 668, 1-97. [CrossRef]

5. Ali, A.; Lange, J.S.; Stone, S. Exotics: Heavy pentaquarks and tetraquarks. Prog. Part. Nucl. Phys. 2017, 97, 123-198. [CrossRef]

6. Olsen, S.L.; Skwarnicki, T.; Zieminska, D. Nonstandard heavy mesons and baryons: Experimental evidence. Rev. Mod. Phys. 2018, 90, 015003. [CrossRef]

7. Makhaldiani, N. Phase transitions in the extended particle systems, Hagedorn temperature and fractal dimension of space, as a confinement phase transition order parameter. EPJ Web Conf. 2019, 204, 06012. [CrossRef]

8. Kanwal, R.P. Generalized Functions Theory and Technique, 2nd ed.; Birkhauser: Boston, MA, USA; Basel, Switzerlands; Berlin, Germany, 1998.

9. Bali, G. QCD forces and heavy quark bound states. Phys. Rept. 2001, 343, 1-136. [CrossRef]

10. Makhaldiani, N. Fractal Calculus (H) and some Applications. Phys. Part. Nuclei Lett. 2011, 8, 535-544. [CrossRef]

11. Isaev, A.P. Multi-loop Feynman Integrals and Conformal Quantum Mechanics. Nucl. Phys. B 2003, 662, 461-475.

12. Makhaldiani, N. New Hamiltonization of the Schrödinger Equation by Corresponding Nonlinear Equation for the Potential; JINR-E-2-2000-179; Joint Institute for Nuclear Research (JINR): Dubna, Russia, 2000.

13. Debye, P.; Hückel, E. The theory of electrolytes. 1. Freezing point depression and related phenomena. Phys. Z. 1923, 24, 185-206. [Translated by Michael J. Braus (2019).]

14. Dixit, V.V. Charge Screening and Space Dimension. Mod. Phys. Lett. 1990, 5, 227-235. [CrossRef]

15. Kharzeev, D.E.; Levin, E.M. Color confinement and screening in the $\theta$-vacuum. Phys. Rev. Lett. 2015, 114, 242001. [CrossRef] [PubMed]

16. Di Vecchia, P.; Fabricius, K.; Rossi, G.; Veneziano, G. Preliminary evidence for $U_{A}(1)$ breaking in QCD from lattice calculations. Nucl. Phys. B 1981, 192, 392-408. [CrossRef]

17. Makhaldiani, N.; Müller-Preussker, M. The topological susceptibility from SU(3) lattice gauge theory. JETP Lett. 1983, 37, 523-526.

18. Fabricius, K.; Rossi, G. Monte Carlo measurement of the topological susceptibility in SU(3) lattice gauge theory. Phys. Lett. B 1983, 127, 229-232. [CrossRef]

19. Muller-Preussker, M. Recent results on topology on the lattice (in memory of Pierre van Baal). arXiv 2015, arXiv:1503.01254.

20. Mumford, D. Tata Lectures on Theta, I; Springer-Modern Birkhäuser Classics: Basel, Switzerland; Boston, MA, USA, 2007.

(C) 2020 by the authors. Licensee MDPI, Basel, Switzerland. This article is an open access article distributed under the terms and conditions of the Creative Commons Attribution (CC BY) license (http:/ / creativecommons.org/licenses/by/4.0/). 